\title{
Analytical Method for Screw Rotor Cutting according to the Cutter Workpiece Engagement Model
}

\author{
Arifin Achmad ${ }^{1}$ and Yu-Ren $\mathrm{Wu}^{2, *}$ \\ ${ }^{1}$ Department of Mechanical Engineering Education, Universitas Negeri Yogyakarta, 01, Colombo Rd, \\ Karangmalang, Yogyakarta 55281, Indonesia \\ ${ }^{2}$ Department of Mechanical Engineering, National Central University, 300, Zhongda Rd., Zhongli District, \\ Taoyuan City 320317, Taiwan (R.O.C.)
}

\begin{abstract}
The proper screw rotor cutting process is essential to obtain a precise rotor profile; however, it is a costly and high hazard if studied in a practical method. This study introduces an analytical screw cutting method to ensure the cutting process is running well and acquire the expected rotor profile. Two distinct cutter types, a cutter with a single curve and multiple inserts cutting edge, were applied. The analytical screw cutting method was developed according to the cutter-workpiece engagement model. The result reveals that the analytical screw cutting model using various cutters can generate identical simulated profiles and close to the original rotor profile. In addition, the virtual machining verification using VERICUT software was conducted to evaluate the proposed method. Conclusively, the analytical screw cutting method is reliable and realistic to be applied in screw rotor milling.
\end{abstract}

Keywords: Screw rotor machining, Disk-type milling cutter, Inserts cutting trajectory, Cutter workpiece engagement, Analytical screw cutting.

\section{INTRODUCTION}

A screw rotor has a particular form, like a threaded cylindrical workpiece applied on a broader field of industrial function. Many screw rotor manufacturers only consider two rotor machining processes: roughing by milling and finishing by grinding [1]. Study on rotor machining is high cost and risk if performed in a practical method. Hence, it is necessary to introduce an analytical method to simulate the screw cutting process to enhance cost-time saving in rotor manufacturing.

Screw rotor cutting can be illustrated as a dynamic machining process where the engagement among cutter cutting edge and the in-process workpiece surface is a continuous instant cutting process. In addition, screw rotor precision milling constitutes a roughing method to form rotor profiles. The triangle mesh model [2] and the instantaneous cutting flute and exit-entry [3] were studied to describe it. It can also be assumed to be generating machining that employs a two-parameter enveloping theory, such as machined profile calculation and instant contact analysis [4, 5]. In addition, a cutting simulation of the grinding process can be adapted to screw rotor cutting using form milling cutter. Zhao et al. [6] proposed a mathematical model of screw rotor precision grinding using a computer numerical control (CNC) grinding wheel dressing. Wu and Fan [7] introduced a mathematical model for a cutting simulation of screw rotor form grinding on a vertical multi-axis CNC machine. Screw milling cutter

Address correspondence to this article at the Department of Mechanical Engineering, National Central University, 300, Zhongda Rd., Zhongli District, Taoyuan City 320317, Taiwan (R.O.C.); Tel: 886-3-4227151 ext. 34332; Fax: 886-3-4224501; E-mail: yurenwu@ncu.edu.tw profile has a high impact on the machined rotor profile accuracy, where it can be generated according to a pair meshing among the screw rotor and the cutter wheel [8-11]. A mathematical model was introduced to arrange multiple inserts on an end-milling cutter body $[12,13]$ and a universal multi-insert profile cutter [14]. In addition, multiple insert arrangement on form milling cutter was studied [11].

This paper introduces an analytical method of screw rotor cutting to ensure the cutting process is running well and acquire the expected rotor profile. In addition, it can enhance the cost-time saving in rotor manufacturing. The cutting simulation is performed using two distinct cutter types, a cutter with a single curve and multiple inserts cutting edge. Firstly, the cutter-workpiece engagement concept is applied to describe the cutting edge movement of the milling cutter and construct the relative motion of CNC machine axes. Then, a cutter stock curve as a single cutting edge is generated according to the pair meshing of the screw rotor and the cutter wheel body. Furthermore, a cutter with multiple inserts cutting edge is established. Ultimately, numerical examples and the discussion are demonstrated to evaluate the correctness of the analytical screw cutting method application, including virtual machining verification.

\section{METHODOLOGY}

This paper utilizes an analytical approach that is cutter workpiece engagement model to represent the motion relationship among rotor and cutter wheel according to a meshing condition and gear enveloping theory; then, the motion relation is applied on the axes movement of CNC machine for screw rotor cutting 
process. In addition, according to the meshing condition, the cutter profile is generated that will be applied as a boundary area of insert arrangement. Insert position arrangement onto cutter body also studied by analytical method.

\subsection{Cutter Workpiece Engagement Model}

Cutter-workpiece engagement (CWE) is an essential role in machining process simulation that illustrates an instantaneous contact geometry amongst the cutter cutting edge and the in-process workpiece during the machining process $[2,3]$. It can be applied to describe a screw rotor cutting simulation using a disk-type milling cutter with multiple inserts where the rotor profile is estimated by the inserts cutting trajectory onto the workpiece cut surface. The screw rotor cutting process can be illustrated thoroughly as a single path of insert cutting edge when removing a stock material along the cutting process, as shown in Figure 1(a). Since the insert is installed on the cutter body, it will travel simultaneously in rotates around the cutter axis while additionally moving straight in respect to the workpiece rotary axis that according to the three principal movements simultaneously inter-machine axes, i.e., the milling cutter revolution, the workpiece rotation, and the axial translation of milling cutter. The relation among the three is that the inter-connection among cutter translation travels along the workpiece rotary axis. The prescribed feeding speed controls the workpiece rotational speed, while the milling cutter also independently revolves in its rotary axis, as illustrated in Figure 1(b). Finally, multiple insert cutting edges will remove the stock material sequentially according to its position on the cutter body; further, a generated tooth surface profile of the screw rotor can be obtained.

The inter-machine axes movement of the CNC rotor milling machine model is set to the prevailing movement relation of the cutter-workpiece instant contact as addressed before then utilized to perform the screw rotor cutting simulation. Figure 2 illustrates the coordinate system of the CNC rotor machine and its motion relation, where the machine motion diagram is assumed to have three rotary axes, A, B, and C, namely workpiece rotary axis, cutter head rotary axis, and milling cutter spindle, respectively. Moreover, it is assumed to have three sliding axes, $\mathrm{X}, \mathrm{Y}$, and $\mathrm{Z}$, radial sliding axis, tangential sliding axis, and axial sliding axis, respectively, where the axes are concerning the workpiece. The cutting movement in the CNC rotor machine model is represented in a transformation matrix according to the workpiece rotational angle, as derived in Eq. (1), whereas the inter-machine axes relation can then be described as a function of the motion parameter, as expressed in Eq. (2).

$\mathbf{M}_{j f}=\left[\begin{array}{cccc}\cos \varphi_{c} & -\sin \varphi_{c} & 0 & 0 \\ \sin \varphi_{c} & \cos \varphi_{c} & 0 & 0 \\ 0 & 0 & 1 & 0 \\ 0 & 0 & 0 & 1\end{array}\right]\left[\begin{array}{cccc}1 & 0 & 0 & 0 \\ 0 & \cos \varphi_{s} & -\sin \varphi_{s} & 0 \\ 0 & \sin \varphi_{s} & \cos \varphi_{s} & 0 \\ 0 & 0 & 0 & 1\end{array}\right]\left[\begin{array}{llll}1 & 0 & 0 & C_{x} \\ 0 & 1 & 0 & C_{y} \\ 0 & 0 & 1 & C_{z} \\ 0 & 0 & 0 & 1\end{array}\right]\left[\begin{array}{cccc}\cos \varphi_{r} & \sin \varphi_{r} & 0 & 0 \\ -\sin \varphi_{r} & \cos \varphi_{r} & 0 & 0 \\ 0 & 0 & 1 & 0 \\ 0 & 0 & 0 & 1\end{array}\right]$
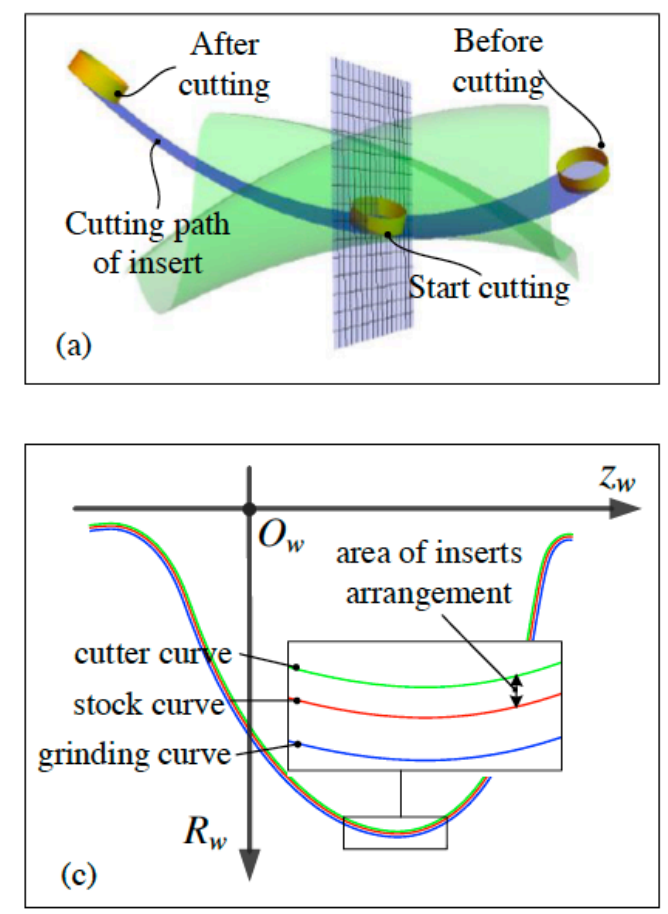

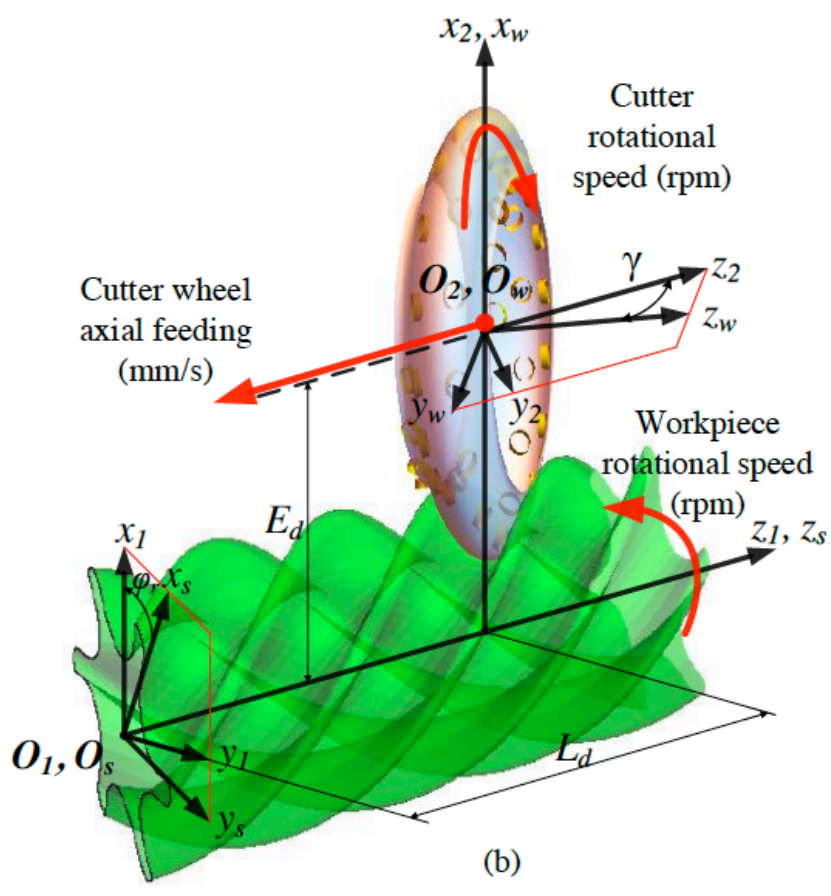

(b)

Figure 1: The cutting principal on screw rotor milling (a) insert cutting trajectory, (b) general coordinate system of the screw rotor - cutter wheel motion relation, (c) generated stock and cutter profile curves. 


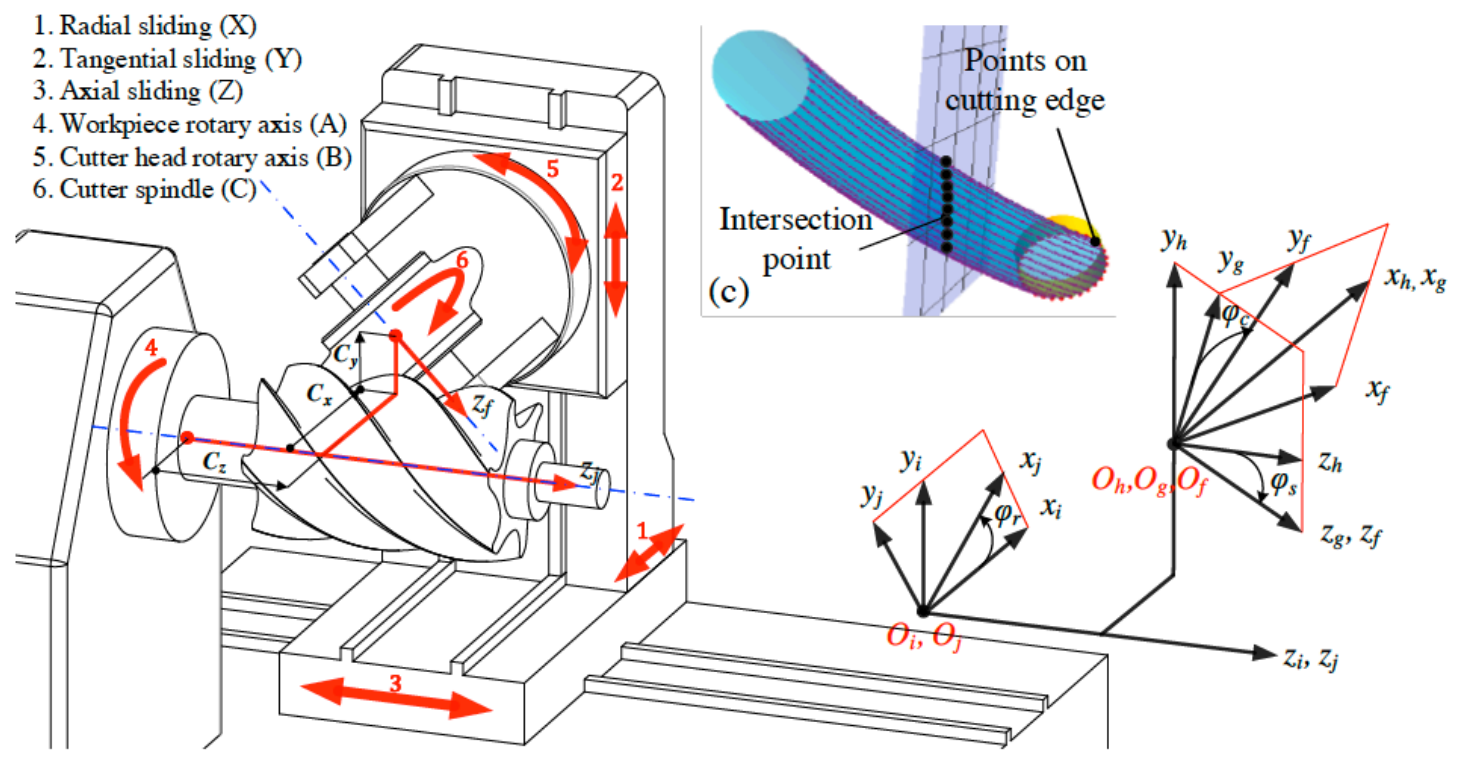

Figure 2: CNC machine model (a) the axes motion relationship, (b) transform coordinate system cutter to workpiece and (c) cutting points by insert trajectory.

$\varphi_{s}=\pi / 2-\gamma ; C_{x}=E_{d} ; C_{y}=0 ; C_{z}\left(\varphi_{r}\right)=s_{p} \varphi_{r}$

\subsection{Generation of Stock Curve as a Cutter Cutting Edge}

The milled rotor possesses an additional dimension compared with the original rotor; it considers a grinding allowance machined in the finishing by grinding process. To obtain the stock cutter cutting edge then the original rotor profile is modified to evolve a rotor stock profile, where the profile curve generation considers combining the following two ideas: a screw rotor revolving with a declination angle [13] and enlarged with a similar allowance on the rotor profile in a normal direction.

When the data point set of the original rotor profile $\mathbf{r}_{0}$ is provided, then Cubic-Spline is applied as a curve fitting method [13], as expressed in Eq. (3). Since the tooth profile generation on the screw rotor machining has potential normal errors, a declination angle in the initial position of rotor profile discrete point data is required to solve it, where the revolved profile $\mathbf{r}_{d}$ with a declination angle $\psi$ is calculated by Eq. (4):

$$
\begin{aligned}
& \mathbf{r}_{0}(u)=\left[x_{0}(u), y_{0}(u)\right]=\left[\sum_{k=0}^{3} C_{k, x} u_{x}^{k}, \sum_{k=0}^{3} C_{k, y} u_{y}^{k}\right], \quad 0 \leq u \leq u_{s} \\
& \mathbf{r}_{d}(u)=\left[x_{d}, y_{d}\right]=\left[x_{0} \cos \psi-y_{0} \sin \psi, x_{0} \sin \psi-y_{0} \cos \psi\right]
\end{aligned}
$$

where $u$ is the fitted curve parameter (rotor profile parameter); $C_{k, x}$ and $C_{k, y}$ are the coefficients of the fitted equations of $x_{0}(u)$ and $y_{0}(u)$, respectively; $k$ is an order of the fitted equation $(k=0 \sim 3)$; and $u_{s}$ is the maximum value of the fitted curve parameter.
When the revolved rotor profile $\mathbf{r}_{d}$ is appended by its unit normal vector $\mathbf{n}_{d}$ with $\mathbf{k}=[0,0,1]$ is a unit vector along the z-axis that is multiplied by grinding allowance $\eta_{r}$, then the enlarged rotor profile $\mathbf{r}_{h}$ can be acquired by deriving Eqs. (5) and (6).

$$
\begin{aligned}
& \mathbf{n}_{d}(u)=\frac{\mathbf{N}_{d}}{\left|\mathbf{N}_{d}\right|}, \mathbf{N}_{d}=\partial_{u}\left[x_{d}, y_{d}, 1\right] \times \mathbf{k} \\
& \mathbf{r}_{h}(u)=\mathbf{r}_{d}(u)+\eta_{r} \mathbf{n}_{d}(u)
\end{aligned}
$$

The enlarged rotor profile $\mathbf{r}_{h}$ is dedicated to be a rotor profile on the transverse plane of the screw rotor, then it is moved by a spiral motion along the rotor axial direction, so that the screw rotor stock surface is shaped. According to the enlarged rotor profile $\mathbf{r}_{h}$, the rotor stock surface $\mathbf{r}_{s}$ and its normal vector $\mathbf{n}_{s}$ are derived as follows:

$\mathbf{r}_{s}\left(u, \theta_{r}\right)=\left[x_{s}, y_{s}, z_{s}, 1\right]=\left[x_{h}(u) \cos \theta_{r}+y_{h}(u) \sin \theta_{r},-x_{h}(u) \sin \theta_{r}+y_{h}(u) \cos \theta_{r}, s_{p} \theta_{r}, 1\right]$

$$
\begin{aligned}
& \mathbf{n}_{s}\left(u, \theta_{r}\right)=\frac{\mathbf{N}_{s}}{\left|\mathbf{N}_{s}\right|}, \mathbf{N}_{s}=\partial_{\theta_{r}} \mathbf{r}_{s}^{\prime} \times \partial_{u} \mathbf{r}_{s}^{\prime} \\
& \text { and } \mathbf{r}_{s}^{\prime}=\left[x_{s}\left(u, \theta_{r}\right), y_{s}\left(u, \theta_{r}\right), s_{p} \theta_{r}\right]
\end{aligned}
$$

where $u$ and $\theta_{r}$ are the surface variables of the rotors; and parameter $s_{p}$ is the rotors unit lead.

Furthermore, the transform motion from rotor coordinate system to cutter wheel coordinate system is established in a general coordinate system according to the relative motion among the screw rotor and cutter wheel as expressed in Eq. (9). The relative spatial position among the rotor and the cutter wheel is defined by the cutter setting angle $\gamma$ and the shortest 
center distance $E_{d}$ among them as shown in Figure 1 (b).

$\mathbf{M}_{w s}=\left[\begin{array}{cccc}1 & 0 & 0 & 0 \\ 0 & \cos \gamma & -\sin \gamma & 0 \\ 0 & \sin \gamma & \cos \gamma & 0 \\ 0 & 0 & 0 & 1\end{array}\right]\left[\begin{array}{cccc}1 & 0 & 0 & -E_{d} \\ 0 & 1 & 0 & 0 \\ 0 & 0 & 1 & -L_{d} \\ 0 & 0 & 0 & 1\end{array}\right]\left[\begin{array}{cccc}\cos \varphi_{r} & -\sin \varphi_{r} & 0 & 0 \\ \sin \varphi_{r} & \cos \varphi_{r} & 0 & 0 \\ 0 & 0 & 1 & 0 \\ 0 & 0 & 0 & 1\end{array}\right]$

where $\varphi_{r}$ is the rotor rotational angle; and $L_{d}=\left(s_{p} \varphi_{r}\right)$ is the cutter wheel translation along the rotor axis.

Subsequently, the contact curve $\mathbf{r}_{w}$ and its normal vector $\mathbf{n}_{w}$ is obtained by transforming the rotor stock surface $\mathbf{r}_{s}$ and its normal vector $\mathbf{n}_{s}$ using the transformation matrix $\mathbf{M}_{w s}$ as follows:

$\mathbf{r}_{w}\left(u, \theta_{r}, \varphi_{r}\right)=\mathbf{M}_{w s}\left(\varphi_{r}\right) \mathbf{r}_{s}\left(u, \theta_{r}\right)$

$\mathbf{n}_{w}\left(u, \theta_{r}, \varphi_{r}\right)=\mathbf{L}_{w s}\left(\varphi_{r}\right) \mathbf{n}_{s}\left(u, \theta_{r}\right)$

where $\mathbf{M}_{w s}$ is the transformation matrix from the rotor motion coordinate system $S_{S}$ to the cutter wheel coordinate system $S_{W}$; and $\mathbf{L}_{w s}$ denotes a submatrix which takes the first $3 \times 3$ element of $\mathbf{M}_{w s}$.

According to the gearing enveloping theory, the meshing equation of the instant contact curve among the screw rotor (the rotor stock surface) and cutter wheel is derived as follows:

$$
f_{w}\left(u, \theta_{r}, \varphi_{r}\right)=\mathbf{n}_{w} \cdot\left(\mathbf{k} \times \mathbf{r}_{w}^{\prime}\right)=0, \quad \mathbf{r}_{w}^{\prime}=\left[x_{w}, \mathrm{y}_{w}, z_{w}\right]
$$

where $\left(\mathbf{k} \times \mathbf{r}_{w}^{\prime}\right)$ means the tangent vector at any points on the cutter wheel profile; and $\mathbf{k}=[0,0,1]$ is the unit vector of the $\mathrm{z}_{\mathrm{w}}$-axis. The longitudinal parameter of the corresponding rotor surface $\theta_{y}$ is acquired by assuming the rotor in constant position with rotational angle $\varphi_{r}=0$ and substituting the rotor profile parameter $u$ with the value that was previously obtained by using Cubic-Spline. Then, all acquired parameter values are applied to solve Eq. (10). Finally, the instant contact position among the screw rotor and cutter wheel at the particular position is acquired.

The cutter stock curve is achieved by projecting the contact curve to a plane perpendicular to the cutter wheel rotational axis. The axial-section profile of the cutter stock curve at the cutter coordinate system is constructed by deriving an equation for the discrete points in the axial-section profile of the cutter stock curve $\mathbf{r}_{t}$ as defined in Eq. (13), where $R_{w}$ and $z_{w}$ are the coordinate values in the axial-section profile of the cutter stock curve.

$$
\mathbf{r}_{t}\left(u, \theta_{r},(u)\right)=\left[R_{w}, z_{w}, 1\right], \quad R_{w}=\sqrt{\left(x_{w}\right)^{2}+\left(y_{w}\right)^{2}}
$$

The curve profile of milling cutter body $\mathbf{r}_{c}$ is determined by appending the cutter stock curve with a normal-shift distance, which is obtained by multiplying the unit normal vector of the cutter stock curve $\mathbf{n}_{t}$ with a normal-shift distance $\eta_{c}$. In addition, the unit normal vector of the stock curve $\mathbf{n}_{t}$ and the milling cutter curve profile $\mathbf{r}_{c}$ are derived in Eqs. (14) and (15), respectively.

$$
\begin{aligned}
& \mathbf{n}_{t}(u)=\frac{\mathbf{N}_{t}}{\left|\mathbf{N}_{t}\right|}, \mathbf{N}_{t}=\partial_{u} \mathbf{r}_{t} \times \mathbf{k}, \text { and } \mathbf{k}=[0,0,1] \\
& \mathbf{r}_{c}(u)=\mathbf{r}_{t}(u)+\eta_{c} \mathbf{n}_{t}(u)
\end{aligned}
$$

Finally, the cutter stock curve is employed as the imaginary profile of outer boundary edge for insert position arrangement; whereas, the cutter profile curve is assumed as a physical boundary of the cutter wheel body. The distance among both curves will be applied to install the inserts by considering several position variables.

\subsection{Establishment of Multiple Inserts Cutting Edge}

The multiple inserts are established onto cutter body according to the explicit geometric relations among the insert cutting edge, the cutter stock curve, and the cutter wheel body curve on the axial plane of the cutter. Firstly, the local coordinate system of the insert cutting edge is defined by considering several space variables as shown in Figure 3, where the insert inclination angle is determined according to practical machining experience. Then the cutter stock and body wheel curves are divided into four segments: (1) right segment with sharp curvature; (2) right segment with slight curvature; (3) left segment with sharp curvature; and (4) left segment with slight curvature. The standard round type insert is distributed mathematically to each segment by projecting the insert cutting edge from the local coordinate system onto the milling cutter body profile on the axial plane. Finally, the insert position is projected from the axial plane onto the $3 \mathrm{D}$ position distribution on the whole cutter body by considering position adjustment with correctional offset and any index angle.

The insert cutting edge is defined by the radius $r$ on the $\left(x_{e}, y_{e}\right)$ plane in the counter-clockwise direction from local axis $\left(x_{e}\right)$, where $\sigma$ is the insert curve parameter, as expressed in Eq. (16):

$\mathbf{r}_{e}(\sigma)=\left[x_{e}, y_{e}, z_{e}, 1\right]^{T}=[r \cos \sigma, r \sin \sigma,-t, 1]^{T}$

The space variables of the insert position consist of two offsets and three position angles, which are as follows: axial offset $Z_{4}$; radial offset $R_{4}$; rotational 


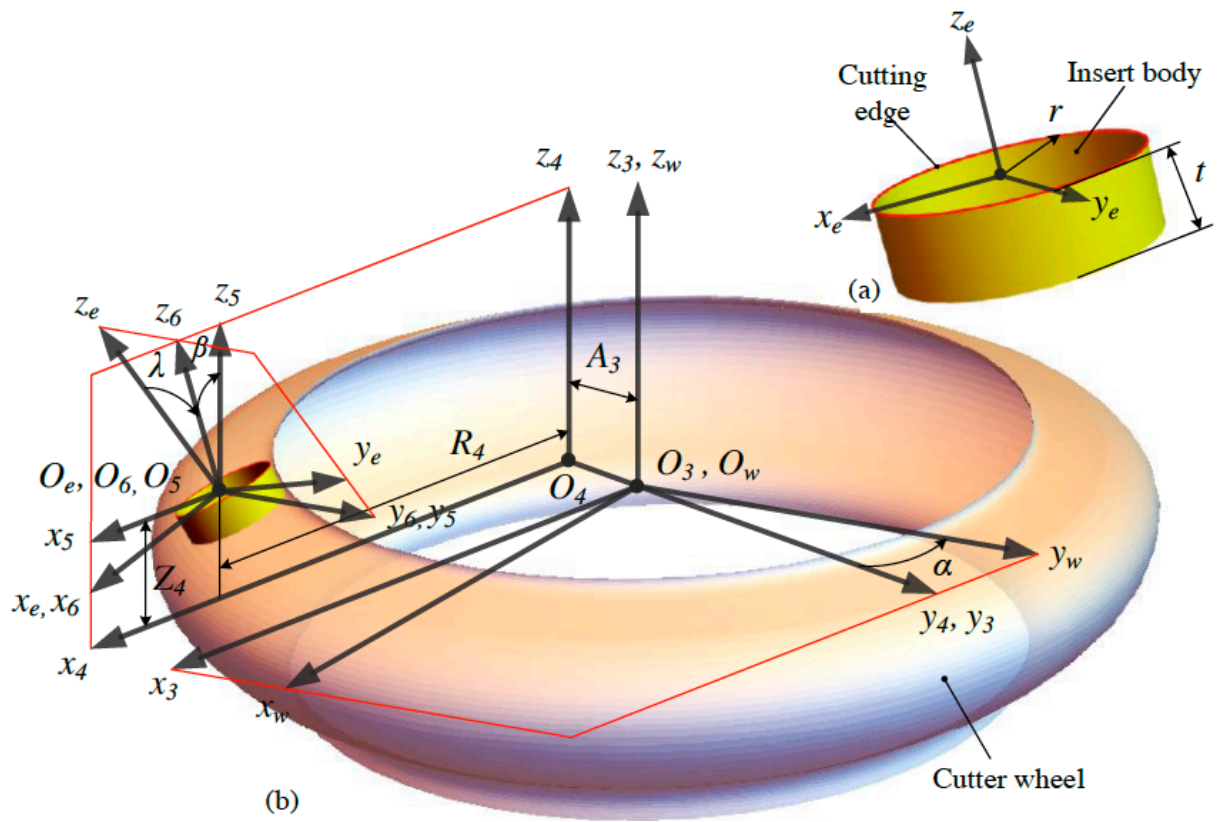

Figure 3: Insert position establishment according to (a) round insert cutting edge profile and (b) local coordinate system of the cutter wheel.

angle $\beta$, which rotates around the $y_{5}$-axis; inclination angle $\lambda$, which rotates around the $x_{6}$-axis; and index angle $\alpha$, which rotates around the $z_{w}$-axis. There is one additional variable, which is correctional offset $A_{3}$ on the $y_{3}$-axis that is perpendicular to radial offset $R_{4}$. The distribution of the multiple inserts onto the cutter body is completed by solving all of the variables in Eq. (17), where $\mathbf{M}_{w e}$ is the transformation matrix from the insert coordinate system $S_{e}$ to the milling cutter coordinate system $S_{w}$ :

$\mathbf{r}_{e}^{(w)}=\left[\begin{array}{c}x_{e}^{(w)} \\ y_{e}^{(w)} \\ z_{e}^{(w)} \\ 1\end{array}\right]=\mathbf{M}_{w e} \mathbf{r}_{e}$

where

$\mathbf{M}_{w e}=\left[\begin{array}{cccc}\cos \alpha & -\sin \alpha & 0 & 0 \\ \sin \alpha & \cos \alpha & 0 & 0 \\ 0 & 0 & 1 & 0 \\ 0 & 0 & 0 & 1\end{array}\right]\left[\begin{array}{cccc}1 & 0 & 0 & R_{4} \\ 0 & 1 & 0 & -A_{3} \\ 0 & 0 & 1 & Z_{4} \\ 0 & 0 & 0 & 1\end{array}\right]\left[\begin{array}{cccc}\cos \beta & 0 & \sin \beta & 0 \\ 0 & 1 & 0 & 0 \\ \sin \beta & 0 & \cos \beta & 0 \\ 0 & 0 & 0 & 1\end{array}\right]\left[\begin{array}{cccc}1 & 0 & 0 & 0 \\ 0 & \cos \lambda & -\sin \lambda & 0 \\ 0 & \sin \lambda & \cos \lambda & 0 \\ 0 & 0 & 0 & 1\end{array}\right]$

Then the technical step of insert arrangement is conducted with the following stages: preparation, arranging on the sharp curvature curve, arranging on the slight curvature curve, adjustment to the 3D position by a correctional offset, and distributing to the cutter chip grooves.

\section{NUMERICAL EXAMPLE AND DISCUSSION}

The analytical screw cutting simulation is demonstrated according to the data set of female screw rotor as the original profile, provided by Hanbell Company Ltd. Table $\mathbf{1}$ presents the geometric parameters of the female screw rotor and the milling cutter.

Table 1: Geometric Parameter of Screw Rotors and Milling Cutter

\begin{tabular}{|c|c|c|}
\hline Items (unit) & Symbols & Female Rotor \\
\hline \hline Tooth number & $\mathrm{N}$ & 6 \\
\hline Declination angle $\left(^{\circ}\right)$ & $\mu$ & 15.0 \\
\hline Pitch helix angle $\left(^{\circ}\right)$ & $\phi_{2}$ & 46.0 \\
\hline $\begin{array}{c}\text { Datum center distance among } \\
\text { rotors (mm) }\end{array}$ & $C_{d}$ & 123.0 \\
\hline Rotor length (mm) & $L$ & 150.5 \\
\hline Normal-shift of rotor (mm) & $\eta_{r}$ & 0.40 \\
\hline Rotor outer diameter (mm) & $d_{a}$ & 138.99 \\
\hline Setting angle $\left({ }^{\circ}\right)$ & $\gamma$ & 44.0 \\
\hline Wheel outer diameter (mm) & $d_{i}$ & 206.83 \\
\hline Normal-shift of cutter (mm) & $\eta_{c}$ & 0.075 \\
\hline $\begin{array}{c}\text { Datum center distance among } \\
\text { wheel and rotors (mm) }\end{array}$ & $E_{d}$ & 139.495 \\
\hline $\begin{array}{c}\text { No. of cutter flute } \\
\text { No. of chip groove }\end{array}$ & $\mathrm{F}$ & 1 \\
\hline & $\mathrm{G}$ & 16 \\
\hline
\end{tabular}

\subsection{Analytical Screw Cutting Applying the Cutter Stock Curve as a Single Cutting Edge}

This section performs the analytical screw cutting using the cutter stock curve as single cutting edge. The 

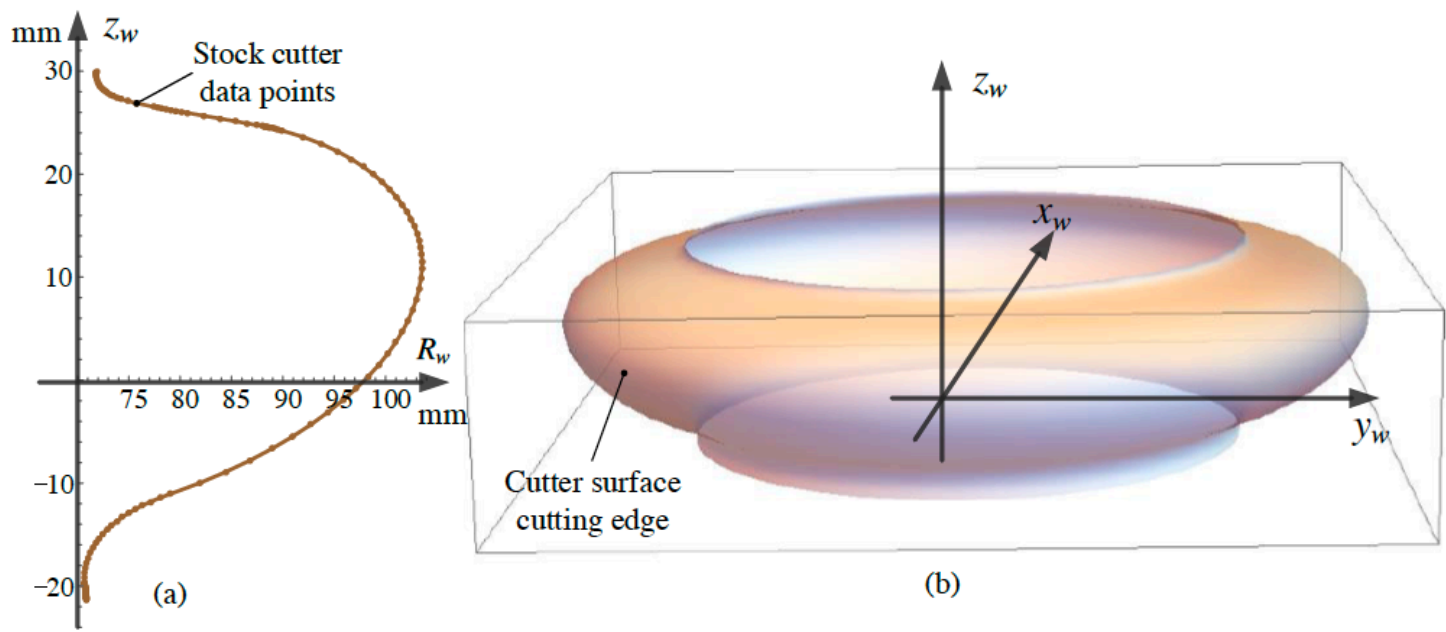

(b)

Figure 4: Cutter model with single cutting edge (a) Stock cutter data point set, (b) stock cutter surface body as the cutting edge.

cutter stock curve was generated according to the method in Section 2.2. and considering the geometric parameter data in Table 1, as shown in Figure 4(a). Then the cutter surface cutting edge was constructed by considering a radial surface parameter $\varphi_{m}$ and revolving the generated point data of the cutter stock curve on cutter rotary axis according to the revolution matrix as derived in Eq. (18) and the resulted cutter with single cutting edge is shown in Figure $\mathbf{4}(\mathbf{b})$.

$\mathbf{r}_{m}\left(u, \varphi_{m}\right)=\mathbf{M}_{m w} \mathbf{r}_{t}$

where

$\mathbf{M}_{m w}=\left[\begin{array}{cccc}\cos \varphi_{m} & \sin \varphi_{m} & 0 & 0 \\ -\sin \varphi_{m} & \cos \varphi_{m} & 0 & 0 \\ 0 & 0 & 1 & 0 \\ 0 & 0 & 0 & 1\end{array}\right]\left[\begin{array}{cccc}1 & 0 & 0 & 0 \\ 0 & \cos (\pi / 2) & -\sin (\pi / 2) & 0 \\ 0 & \sin (\pi / 2) & \cos (\pi / 2) & 0 \\ 0 & 0 & 0 & 1\end{array}\right]$

Furthermore, a screw cutting simulation was performed applying the CNC machine model including the axes motion relation (Figure 2), in which the cutter stock surface was applied as a single cutting edge. The screw cutting process along the rotor length $L$ on one tooth groove was conducted by solving Eqs. (10)-(13) by considering the actual cutting condition; cutter spindle rotational speed $S_{c}=200 \mathrm{rpm}$ and the feeding speed $f_{s}=275 \mathrm{~mm} / \mathrm{min}$. Meanwhile, the depth of cut is equal to rotor groove depth since it is a one step cutting process on rotor tooth groove. Since the transform direction is from cutter to workpiece so that it requires to substitute the rotor stock surface $\mathbf{r}_{s}$ to the cutter surface cutting edge $\mathbf{r}_{m}$ and the general coordinate system matrix $\mathbf{M}_{w s}$ to CNC machine coordinate system matrix $\mathbf{M}_{j f}$ where $\varphi_{c}=0$.

Subsequently, an instantaneous contact line that represents cutter-workpiece engagement when the cutter wheel cuts the rotor surface was obtained, as shown in Figure 5(a). Moreover, Figure 5(b) indicates the rotor tooth surface profile that was acquired by solving the meshing angle on several sections along a particular rotor length. The simulated rotor profile was acquired from one section of the rotor surface profile as displayed in Figure 5(c) on the red curve, whereas the green curve is the original rotor profile. The distance among both curves represents the grinding allowance that was set in the initial process when generating the rotor stock profile. Both profiles of simulated and the original rotor are similar in along rotor profile length. It confirms that the cutter profile is a high precision cutting edge. This result indicates that an analytical screw cutting model applying cutter stock surface as a single cutting edge is practicable.

\subsection{Analytical Screw Cutting Applying the Multiple Inserts Cutting Edge}

This section demonstrates the analytical screw cutting using the disk-type milling cutter with multiple inserts cutting edge. Firstly, the multiple inserts were installed onto the cutter body according to the method in Section 2.3. the standard round type insert was applied, with the corresponding segment parameters being Sandvik Coromant R3001240 MM Grade 2030 Carbide Milling Insert with $11.99 \mathrm{~mm}$ and $3.66 \mathrm{~mm}$ in diameter and thickness, respectively [15], where the insert inclination angle was specified $7^{\circ}$. The total number of inserts is 43 , where Figure $\mathbf{6}(\mathbf{a})$ represents the precisely position of insert cutting edge in the axial plane. Combining of each insert cutting edge shaped the cutter profile that will be applied as the cutter cutting edge to cut the stock workpiece. Meanwhile the analytical 3D model of the mlling cutter with a single flute and sixteen chips grooves is exhibited in Figure 6(b). Both figures of inserts position reveal that the analytical approach on multiple inserts arrangenment 


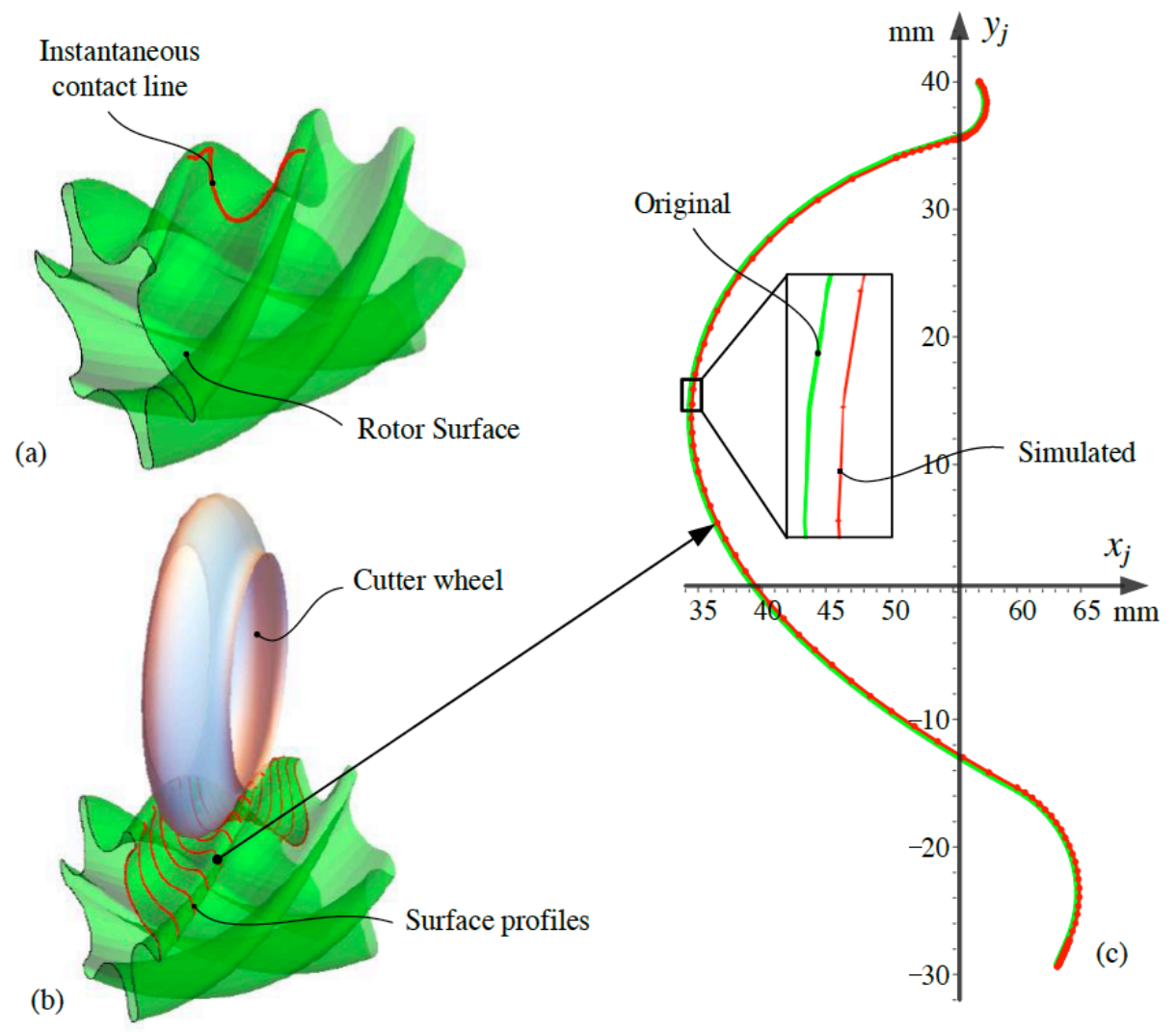

Figure 5: Generated rotor profile by single cutting edge (a) instantaneous contact line among rotor and cutter body, (b) surface rotor profile, and (c) data point set of generated rotor profile.

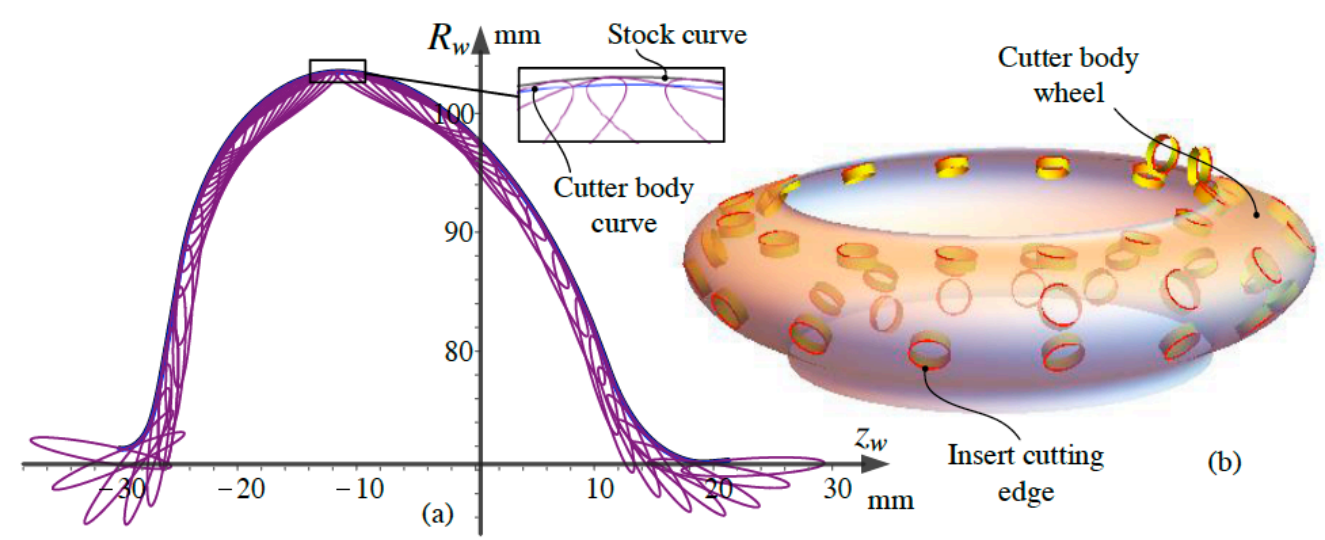

Figure 6: Cutter design with multiple round standard insert (a) insert cutting edge position on axial plane of cutter (b) insert position result on 3D model.

can achieve precisely installation of inserts cutting edge.

Then the cutter and stock workpiece were set in designed relative motion according to the CNC machine model coordinate system as illustrated in Figure 2. The cutting process simulation of the screw rotor in a particular length was performed according to the actual cutting condition; cutter wheel rotational speed $S_{c}=200 \mathrm{rpm}$, the feeding speed $f_{s}=275$ $\mathrm{mm} / \mathrm{min}$, and the depth of cut is equal to the tooth groove depth. The cutting trajectory of multiple inserts cutting edge was calculated by considering all of the motion intercourse on the CNC machine using the angular and linear velocity relationship principle, as derived in Eq. (19).

$\mathbf{r}_{e}^{(j)}\left(\varphi_{c}, \varphi_{r}, \sigma\right)=\left[\begin{array}{c}x_{e}^{(j)} \\ y_{e}^{(j)} \\ z_{e}^{(j)} \\ 1\end{array}\right]=\mathbf{M}_{j f}\left(\varphi_{c}, \varphi_{r}\right) \mathbf{M}_{w e} \mathbf{r}_{e}(\sigma)$

Furthermore, a single transverse plane within the observed rotor length was determined, and a simulated cutting path was obtained according to the intersection 
point among the insert trajectory and the plane, as shown Figure $7(\mathbf{a})$, by solving Eq. (20), where $\left(s_{p} \varphi_{r}\right)$ is the plane position on the rotor length.

$$
\mathbf{r}_{e p}\left(\varphi_{c}, \varphi_{r}\right)=\mathbf{r}_{e}^{(j)}-s_{p} \varphi_{r}=0
$$

Then, by removing the existing points and leaving the adjacent points to the rotor profile, the simulated rotor profile on the transverse plane was obtained, as exhibited in Figure $\mathbf{7}(\mathbf{b})$ on the red curve, whereas the green curve is the original rotor profile and the distance among both curves is represent the grinding allowance. Figure $\mathbf{7 ( b )}$ correspondingly indicates that both the simulated rotor profile and the original rotor profile are similar. This result proves that analytical screw cutting model applying disk-type milling cutter with multiple inserts cutting edge is practicable.
Figure 8 presents the generated rotor profile of both analytical screw cutting models using a cutter with a single curve cutting edge and multiple inserts cutting edge. The blue curve indicates the simulated profile by cutter stock curve with a single cutting edge; conversely, the red curve represents the simulated profile by the cutter with multiple inserts cutting edge. The green curve denotes the original rotor profile as the datum as the comparison control. Although this paper was not evaluate the deviation value among the simulated to the original rotor profile, however, both simulated curves indicate that the analytical screw cutting model using distinct cutters can generate an identical profile. This result confirms that the analytical screw cutting model is reliable and realistic to be applied in screw rotor milling.

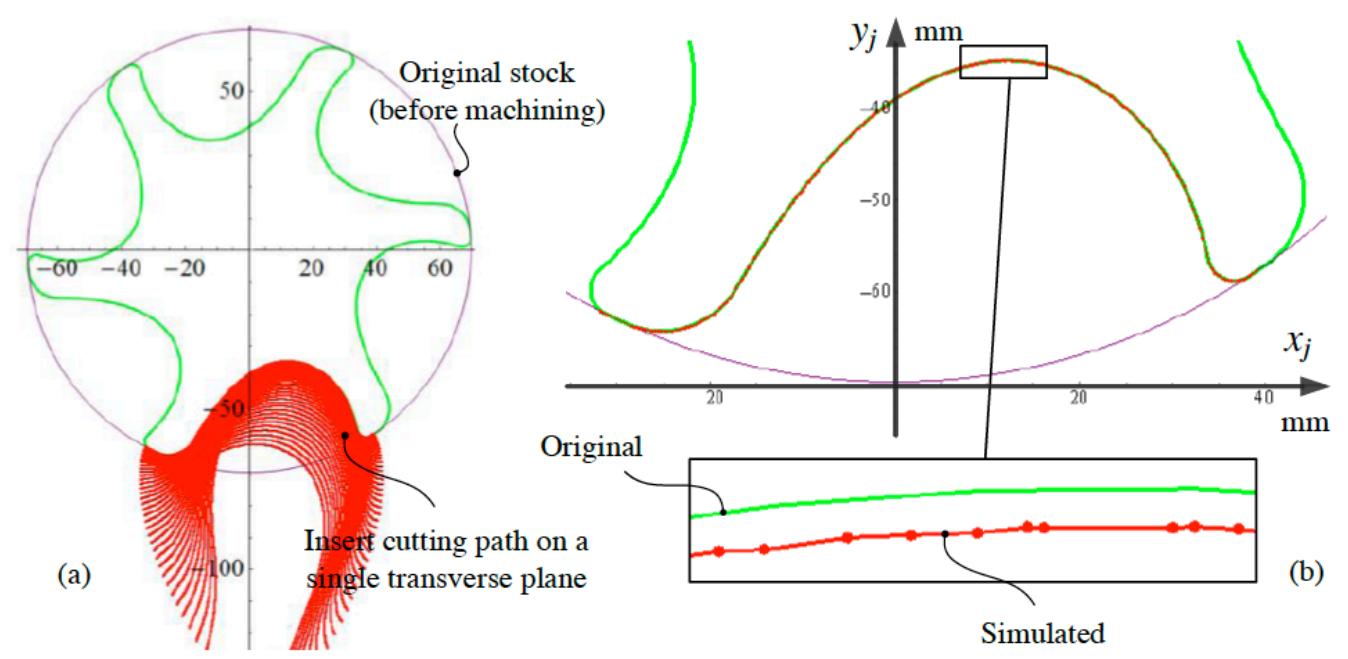

Figure 7: Generated rotor profile by multiple insert cutting edges (a) insert cutting trajectory on a single transverse plane (b) data point set of generated rotor profile.

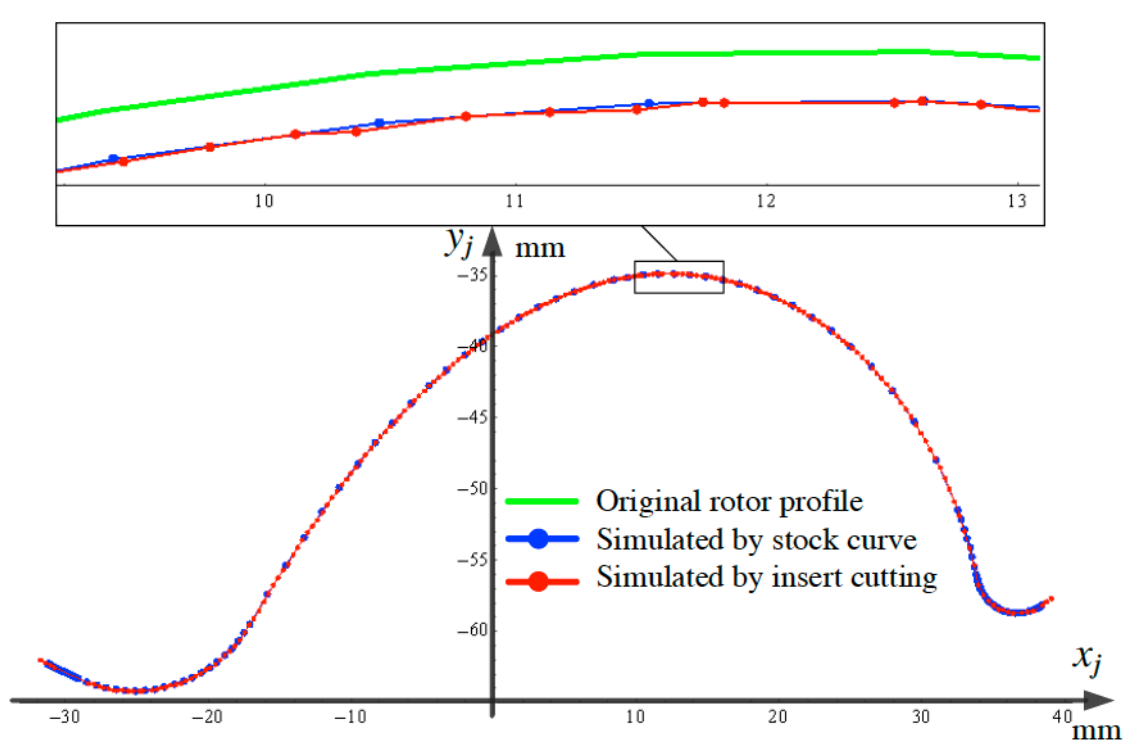

Figure 8: Rotor profile comparison among original, simulated by stock curve and generated by insert cutting edge. 

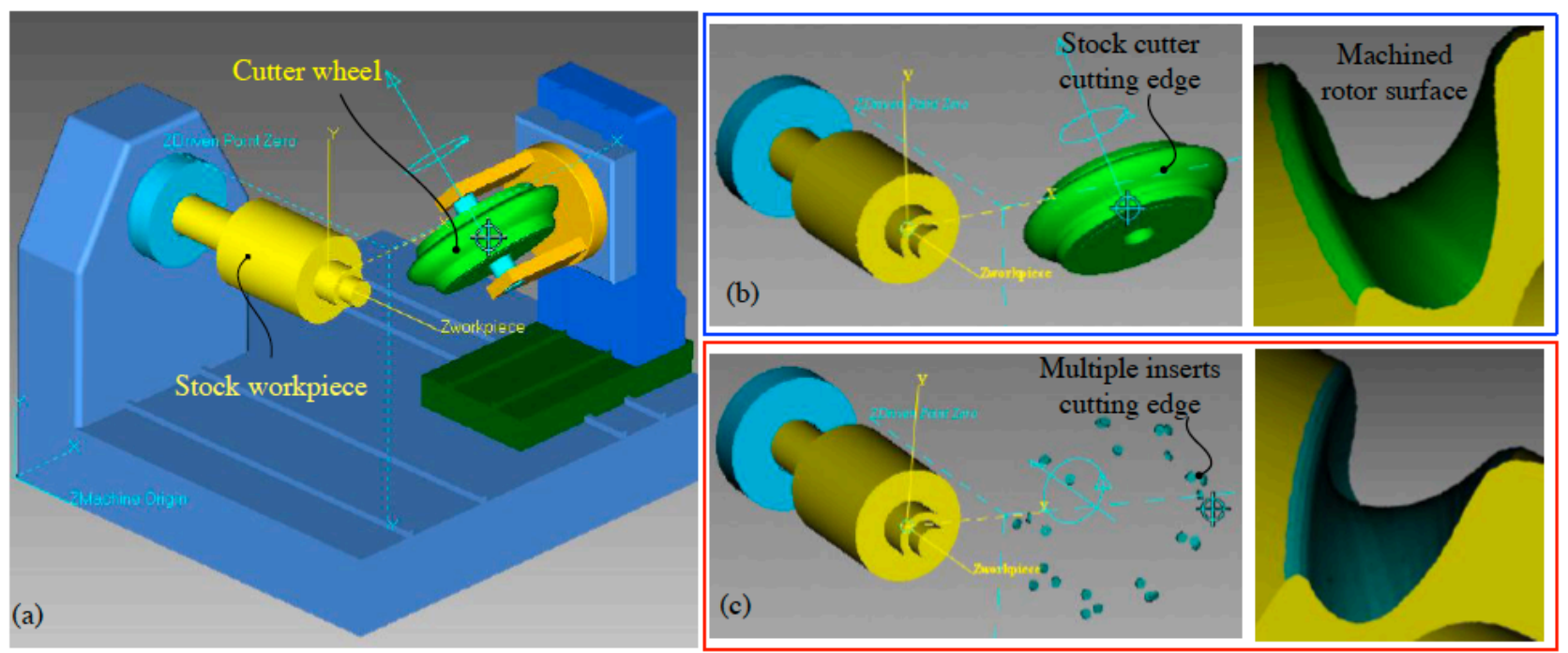

Figure 9: Virtual machining verification using VERICUT software (a) model of the CNC rotor machine, (b) cutting illustration applying cutter with single cutting edge (c) cutter with multiple insert cutting edge.

\subsection{Virtual Machining Verification by VERICUT Software}

This section demonstrates a correctness evaluation using VERICUT software for screw cutting simulation applying two distinct cutter types, a cutter with a single curve cutting edge and multiple inserts cutting edge. Firstly, both cutter types were constructed in completed actual cutter design using Solidworks. Then the geometric model of the CNC machine was established as the virtual environment. Then machine kinematics corresponding to the machine axes relations was evaluated according to Figure 2, subsequent exports the designed actual milling cutter. The kinematics model of the milling cutter and the stock workpiece was established, as illustrated in Figure 9(a). The VERICUT cutting simulation was eventually accomplished with the cutting condition similar to the previous simulation (Section 3.2). Figures $\mathbf{9}(\mathbf{b})$ and $\mathbf{9}(\mathbf{c})$ indicate a cutting specimen of one rotor tooth groove on the stock workpiece by cutter stock and multiple inserts cutting edge, respectively, where it exhibits that both virtual machining can generate an identical profile result. Nevertheless, the deviation of the simulated profile compared to the original rotor was not studied in this paper. The result confirms that the analytical screw cutting model, according to the kinematic relationship on cutter-workpiece engagement, is verified, which can enhance the cost-time saving in rotor manufacturing since the experimental verification is required exclusively one time after being verified by analytical evaluation.

\section{CONCLUSION}

This paper demonstrates an analytical screw cutting method to generate the simulated rotor profile according to the cutter-workpiece engagement model applying two different cutter types with a single curve and multiple inserts cutting edge. Both simulated rotor profile results indicate that the simulation method can generate an identical profile and close to the original rotor profile. The virtual machining verification using VERICUT software proved the proposed method is reliable. Conclusively, the analytical screw cutting method is reliable and realistic to be applied in screw rotor milling, enhancing the cost-time saving in rotor manufacturing since the experimental verification is required exclusively after being verified by analytical evaluation. Hence, it is essential to further research to ensure that the simulated profile deviation is acceptable in industrial applications.

\section{ACKNOWLEDGEMENT}

The authors are grateful to the Ministry of Science and Technology in Taiwan for its financial support under project number MOST 109-2221-E-008004-MY2, and to Hanbell Precise Machinery Co., Ltd. in Taiwan for their technical and financial support.

\section{REFERENCES}

[1] N. Stosic, A geometric approach to calculating tool wear in screw rotor machining, International Journal of Machine Tools \& Manufacture 2006; 46(15): 1961-1965. https://doi.org/10.1016/j.ijmachtools.2006.01.012

[2] X. Gong, and H.Y. Feng, Cutter-workpiece engagement determination for general milling using triangle mesh modeling. Journal of Computational Design and Engineering 2016; 3: 151-160. https://doi.org/10.1016/j.jcde.2015.12.001

[3] S. Lotfi, B. Rami, B. Maher, D. Giles, and B. Wassila, Cutter-workpiece engagement calculation in 3-axis ball end milling considering cutter runout. Journal of Manufacturing Processes 2019; 41: 74-82. https://doi.org/10.1016/j.jmapro.2019.03.025

[4] Y.R. Wu, and W.H. Hsu, A general mathematical model for continuous generating machining of screw rotors with 
worm-shaped tools. Applied Mathematical Modelling 2014; 38(1): 28-37.

https://doi.org/10.1016/j.apm.2013.05.056

[5] K. Jia, J. Guo, S. Zheng, and J.Hong, A general mathematical model for two-parameter generating machining of involute cylindrical gears, Applied Mathematical Modelling 2019; 75: 37-51.

https://doi.org/10.1016/j.apm.2019.05.021

[6] M. Torta, P. Albertelli, and M. Monno, Surface morphology prediction model for milling operations, The International Journal of Advanced Manufacturing Technology 2020; 106(7-8): 3189-3201

https://doi.org/10.1007/s00170-019-04687-x

[7] P. Wang, S. Zhang, Z. Li, and J. Li, Tool path planning and milling surface simulation for vehicle rear bumper mold, Advances in Mechanical Engineering 2016; 8(3): 1-10. https://doi.org/10.1177/1687814016641569

[8] C.J. Chiang, and Z.H. Fong, Design of form milling cutters with multiple inserts for screw rotors, Mechanism and Machine Theory 2010; 45(11): 1613-1627. https://doi.org/10.1016/j.mechmachtheory.2010.06.012

[9] S. Engin, and Y. Altintas, Mechanics and dynamics of general milling cutters. Part II: inserted cutters, International Journal of Machine Tools \& Manufacture 2001; 41(15): 2213-2231.

https://doi.org/10.1016/S0890-6955(01)00046-3

[10] T. Mikolajczyk, D. Y. Pimenov, C. I. Pruncu, K. Patra, H. Latos, G. Krolczyk, M. Mia, A. Klodowski, and M. K. Gupta, Obtaining Various Shapes of Machined Surface Using a Tool with a Multi-Insert Cutting Edge, Applied Sciences-Basel 2019; 9(5): 1-12. https://doi.org/10.3390/app9050880

[11] P. F. Gilles, F. Monies, and W. Rubio, Optimum orientation of a torus milling cutter: Method to balance the transversal cutting force, International Journal of Machine Tools \& Manufacture 2007; 47(15): 2263-2272. https://doi.org/10.1016/j.jmachtools.2007.06.003

[12] Y.Q. Zhao, S Zhao, W. Wei, and H Hou, Precision grinding of screw rotors using CNC method, International Journal of Advanced Manufacturing Technology 2017; 89(9-12): 2967-2979. https://doi.org/10.1007/s00170-016-9241-9

[13] Y.R. Wu, and C.W. Fan, Mathematical modeling for screw rotor form grinding on vertical multi-axis computerized numerical control form grinder, Journal of Manufacturing Science and Engineering 2013; 135(5): 051020. https://doi.org/10.1115/1.4025339

[14] K. Jia, J. Guo, S. Zheng, and J. Hong, A general mathematical model for two-parameter generating machining of involute cylindrical gears, Applied Mathematical Modelling 2019; 75: 37-51. https://doi.org/10.1016/j.apm.2019.05.021

[15] Sandvik Coromant, Rotating tools [catalogues], (2020) 99. http://cataleap.com/blackhawk-flip/books/Sandvik-Coromant/ Sandvik-Coromant-Rotating-Tools-2020/14/index.html

(c) 2022 Achmad and Wu; Zeal Press.

This is an open access article licensed under the terms of the Creative Commons Attribution Non-Commercial License

(http://creativecommons.org/licenses/by-nc/4.0/), which permits unrestricted, non-commercial use, distribution and reproduction in any medium, provided the work is properly cited. 